\title{
Managed-Care-Vorlage: Erläuterungen zur Position von «Hausärzte Schweiz»
}

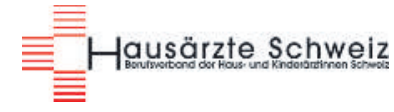

Vorstand Hausärzte Schweiz:

Marc Müller, Präsident

François Héritier,

Vizepräsident

Jürg Rufener, Vizepräsident

Margot Enz Kuhn

Brigitte Zirbs Savigny

Franziska Zogg

Johannes Brühwiler

Gerhard Schilling

Stephan Rupp

Korrespondenz: Hausärzte Schweiz Effingerstrasse 54 Postfach 6052 CH-3001 Bern
Es überrascht wenig, dass nicht alle zufrieden sind mit dem Entscheid der Delegiertenversammlung von «Hausärzte Schweiz». Das Ganze aber gleich als Katastrophe oder Gefährdung der FMH oder noch schlimmer zu werten, erstaunt doch einigermassen. War die Ärzteschaft auch in anderen Fragen jeweils so harmoniebedürftig?

Bei jeder Vorlage gibt es Gegner und Befürworter. Am Schluss bestimmt in einer Demokratie die Mehrheit. Der demokratische Entscheid, auf den es ankommt, ist aber nicht, ob die Ärzteschaft für oder gegen die Managed-Care-Vorlage ist, sondern was das Volk an der Urne entscheiden wird.

Das Schweizer Volk muss entscheiden, weil eine Minderheit der Delegierten in der Ärztekammer nicht zufrieden war mit dem Entscheid der dortigen Mehrheit und deshalb eine Urabstimmung organisiert hat. Bei dieser war nun eine Mehrheit der Minderheit, die überhaupt abgestimmt hat, der Meinung, dass ein Referendum durchgeführt werden müsse, damit die Mehrheit des Volkes möglichst korrigiert, was die Mehrheit der Politiker nach ihrer Meinung «verbockt» hat. Das heisst aber noch lange nicht, dass nun alle nur noch einer Meinung sind.

Innerhalb unseres Berufsverbandes ist der demokratische Prozess zur Entscheidungsfindung genauso abgelaufen, wie es unsere Statuten vorsehen: Die Position des Vorstandes wurde im Lauf der letzten beiden Jahre von der Delegiertenversammlung (DV) insgesamt dreimal bestätigt. Unsere Basis wurde durch verschiedene Artikel in PrimaryCare, Mitgliedermails, Informationen auf unserer Homepage und an zwei Generalversammlungen darüber informiert. Die Delegierten haben im Mai 2011 beschlossen, das Vorliegen der definitiven KVG-Vorlage abzuwarten, bevor sie an einer ausserordentlichen Versammlung einen definitiven Entscheid zum Thema fällen wollten. $\mathrm{Zu}$ deren Vorbereitung haben wir all unsere Mitglieder per Mail und in PrimaryCare aufgefordert, ihren kantonalen Delegierten ihre Meinung mitzuteilen. Die Delegierten wurden dann vom Vorstand mit einer eigentlichen Abstimmungsbroschüre nochmals über Pro und Contra der Vorlage informiert. Nach erneut ausführlicher Diskussion an der DV wurde dann abgestimmt: Mit 31 Ja zu 10 Nein haben unsere Delegierten beschlossen, die Vorlage weiter zu unterstützen. Die Durchführung einer Urabstimmung (Voraussetzung wäre eine Zweidrittelmehrheit) wurde ebenso klar abgelehnt (13:21 Stimmen). Gemäss unseren Statuten besteht nun noch die Möglichkeit, dass 15\% der Mitglieder eine Urabstimmung fordern.

«Hausärzte Schweiz» ist nach wie vor der Meinung, dass die Vorlage mehr Vor- als Nachteile aufweist, auch wenn sie nicht perfekt ist. Sie stärkt die Funktion der Hausärzte in unserem Gesundheitssystem, verbessert den Risikoausgleich und sichert die Unabhängigkeit der Netzwerke von den Versicherern sowie die Vertragsbasis für Integrierte Versorgung. Diese Position zu vertreten, ist legitime Aufgabe unseres Berufsverbands. Sie bedeutet ein Ja zur KVGVorlage und keinen Angriff gegen die FMH.

Nur dadurch ist sichergestellt, dass die Bevölkerung schliesslich in Kenntnis der Vor- und der Nachteile über die Vorlage entscheiden kann («Informed consent»). Wer von Euch wäre zufrieden, wenn er bei einer Volksabstimmung nur die Meinung einer einzigen Partei hören würde, nicht aber diejenige der anderen?

Die FMH hat nur eine reelle Überlebenschance, wenn sie solche demokratisch ausgetragenen Meinungsverschiedenheiten aushält. Denn weit wesentlichere und belastendere Probleme werden erst noch auf sie zukommen: zum Beispiel die Diskussion um die Aufhebung des Kontrahierungszwangs, wenn Managed Care scheitert, oder die Tarifdiskussionen, falls TarVision nicht gelingt. Hier könnten echte Zerreissproben entstehen.

Fazit: Wir gehen davon aus, dass das Referendum zustande kommt. Es wird wie üblich Befürworter und Gegner der Vorlage geben. Wer die Bevölkerung mehr zu überzeugen vermag, wird die Abstimmung gewinnen, und die FMH wird zur Tagesordnung zurückkehren. Das Resultat werden alle akzeptieren und damit leben müssen, mit allen Konsequenzen und verpassten Chancen.

Wir wünschen uns allen die notwendige Gelassenheit und ruhigen Menschenverstand. Überlassen wir die Polemik anderen!

Als Bergbewohner wissen wir, dass das Wasser schliesslich immer abwärts fliesst, auch wenn es zeitweilig Turbulenzen aufweist ...

1 Ärztegesellschaft Baselland. Managed-Care-Vorlage: Basisdemokratie und Hausärzte Schweiz. Schweiz Ärztezeitung. 2011;92(46):1784-5.

2 Bracher D. Offener Brief an den Präsidenten von «Hausärzte Schweiz». Schweiz Ärztezeitung. 2011;92(49):1912. 